\title{
WILEY-VCH
}

DOI: 10.1002/adma.201603074

Article type: Communication

\section{Microfiltration Polymer-based Hollow Fiber Cathode as a Promising Advanced Material for Simultaneous Recovery of Energy and Water}

Krishna P. Katuri*, Narasimha Murthy Srivatsa Bettahalli, Xianbin Wang, Gerald Matar, Stefan Chisca, Suzana Pereira Nunes, and Pascal Elias Saikaly*

Krishna Katuri, Narasimha Murthy Srivatsa Bettahalli, Gerald Matar, Stefan Chisca, Suzana Pereira Nunes, and Pascal Elias Saikaly

Biological and Environmental Sciences and Engineering (BESE) Division, King Abdullah University of Science and Technology, Thuwal 23955-6900, Saudi Arabia

\section{Xianbin Wang}

Advanced Nanofabrication Imaging and Characterization (ANIC) Center, King Abdullah University of Science and Technology, Thuwal 23955-6900, Saudi Arabia

\section{E-mail: pascal.saikaly@kaust.edu.sa; krishna.katuri@kaut.edu.sa}

Keywords: atomic layer deposition, porous hollow fiber cathode, biofouling, resource recovery, membrane bioreactor

Providing adequate supply of clean fresh water and energy as the world's population increases is one of the grand challenges facing society in the current century. ${ }^{[1]}$ One possible solution to address both challenges is to recover clean water for reuse and energy from wastewater by integrating micro- or ultra-filtration membrane cathodes with microbial electrochemical system in what is referred to as electrochemical membrane bioreactor (EMBR). ${ }^{[2]}$ The porous flat cathodes used in EMBR studies served the dual function as the cathode for oxygen reduction reaction (ORR) and membrane for the filtration of treated water. A disadvantage of using porous flat cathodes is their low surface-area-to-volume ratio. Hollow fibers with small radial dimensions provide high surface-area-to-volume ratio, combining compactness, simple and modular reactor design and high performance. ${ }^{[3]}$ Porous inorganic hollow fibers composed of nickel and copper have been used recently as cathodes in aqueous electrolytes for proton or carbon dioxide $\left(\mathrm{CO}_{2}\right)$ reduction. ${ }^{[4,5]}$ Porous polymer-based hollow fibers are flexible, cheaper and easier to manufacture, as well as easier to integrate in modules ${ }^{[6]}$ However, most polymers are electric insulators and there are only a few examples of conducting polymers such as 


\section{WILEY-VCH}

polypyrrole and polyaniline. ${ }^{[7]}$ Processability in solution is a problem, particularly for polypyrrole. ${ }^{[8]}$ Combining high electrical conductivity and catalytic activity with processability as hollow fiber is a challenge.

In this work we propose the use of polymeric hollow fibers as electrodes, prepared from regular non-conductive polymers and coated with a thin electron conductive layer of metal catalyst. However, it is challenging to apply a uniform coating of catalysts on these threedimensional (3D) thin polymeric porous hollow fibers using traditional deposition techniques such as electro-deposition or chemical or physical vapor deposition. ${ }^{[9,10]} \mathrm{A}$ technique, which best meets the requirements for uniform coating and precise catalyst loading on fibers with cylindrical geometry is atomic layer deposition (ALD). ALD is one of the most flexible and powerful deposition techniques currently available due to its exceptional conformality and ability to tune crystallinity, atomic composition and film thickness down to the single-atom level. ${ }^{[11]}$ Because of these advantages, ALD has been applied in various applications including microelectronics, photovoltaics, solid oxide fuel cells, and solar-to-fuel applications, just to name a few. ${ }^{[9-13]}$ To date there have been no reports on the application of ALD on porous polymeric hollow fibers, especially for enabling them to act as electrodes for current collection and catalysis.

Here, we demonstrated for the first time the preparation of electro-catalytic asymmetric porous polymeric hollow fibers by combining the fabrication of porous polymeric hollow fibers made of fluorinated polyoxadiazole (POD) by phase inversion, followed by deposition of very thin layer of platinum $(\mathrm{Pt})$ catalyst on the outer surface by ALD to make the porous POD hollow fibers electro-catalytic (Scheme S1). Phase inversion is easy to upscale and is the most applied method to manufacture polymeric membranes. ${ }^{[14]}$ The asymmetric porous structure promotes high flux with high mechanical stability. The POD hollow fibers demonstrated here could be simply produced by adapting manufacturing processes (i.e. phase inversion), which are already used in the industry ${ }^{[14]}$ At the same time, POD is soluble in organic solvents commonly used 


\section{WILEY-VCH}

for asymmetric membrane manufacture, and hence it is a perfect material to be used in phase inversion hollow fiber manufacture. Most importantly, the high glass transition temperature (> $250{ }^{\circ} \mathrm{C}$ ) of POD makes it ideal for Pt coating by ALD without disturbing the membrane integrity, since the processing temperature within the ALD coating chamber can reach as high as $180{ }^{\circ} \mathrm{C}$ for Pt layer deposition. ${ }^{[15]}$

The electro-catalytic asymmetric porous POD hollow fibers (herein referred to as porous hollow fiber cathodes) is designed for a dual function, i.e. to serve as a microfiltration membrane to allow water to permeate, while the outer surface is electro-catalytic serving as a cathode for hydrogen evolution reaction (HER). Platinum is used here as a model catalyst because of its high electrochemical activity towards HER and to demonstrate the feasibility of this new approach. In addition, the ALD process for Pt deposition is well established. ${ }^{[9,13]}$ In addition, Pt coating serves as an electrode for current collection. In the future, one may explore lower-cost materials for current collection and catalysis. In this study, we focus on integrating the ALD technique into the polymeric membrane fabrication process for the development of complex advanced materials with dual function (i.e. water and energy recovery). This highlights the significance of ALD in creating complex materials for niche specific applications. ${ }^{[1]}$

The cross-section images of the manufactured POD hollow fiber membrane before deposition of Pt by ALD, confirm that the wall thickness is uniform all over the fiber consisting of a mixture of interconnected micro and macro porous structures (Figure S1, Supporting Information). The manufactured POD hollow fiber membranes were subjected to different numbers of ALD platinum cycles (50, 100, 150, 200, 250, 300 and 400). The filtration performance of the porous hollow fiber cathodes as a function of the number of ALD cycles was evaluated by measuring the deionized water permeance (in the form of transmembrane pressure, TMP, required for a fixed water flux) (Figure 1). 


\section{WILEY-VCH}

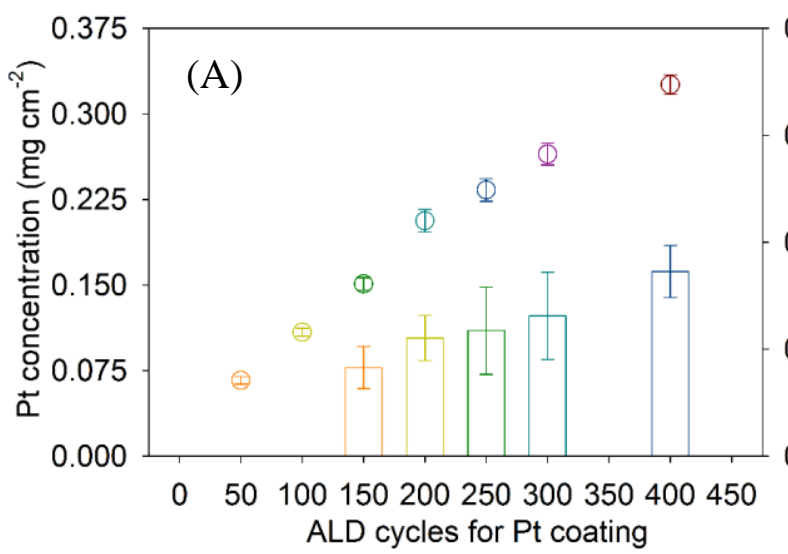

0.020
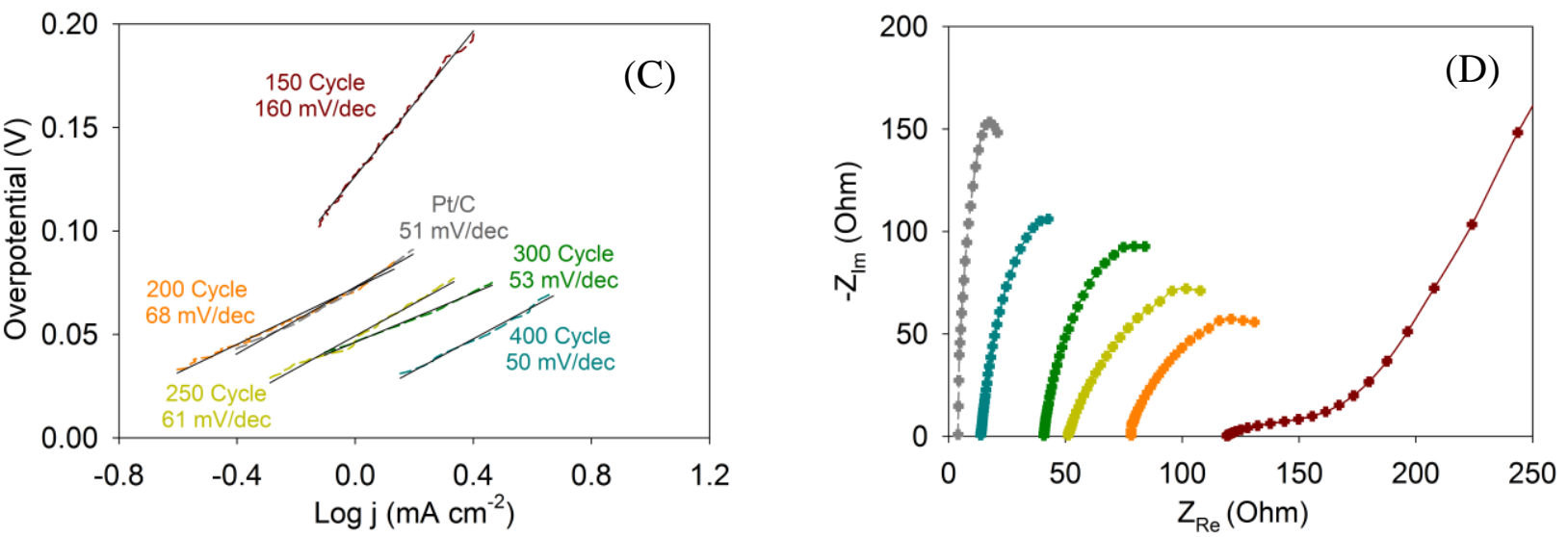

Figure 1. Characteristics of the porous hollow fiber cathodes fabricated with different numbers of ALD platinum cycles on POD hollow fiber membranes. a) Variations in Pt loading (mass of Pt per surface area) on the porous hollow fiber cathodes as a function of the number of ALD cycles (open circles), and its effect on transmembrane pressure (TMP) (vertical bars). TMP was measured using deionized water at a fixed flux $\left(12 \mathrm{~L} \mathrm{~m}^{-2} \mathrm{~h}^{-1}\right)$; b) HER polarization curves $(j-V)$ of porous hollow fiber cathodes prepared at different ALD cycles (150, 200, 250, 300 and 400 cycles) and the results were compared with benchmark Pt-coated carbon cloth cathodes (Pt/C). A $0.5 \mathrm{M} \mathrm{H}_{2} \mathrm{SO}_{4}$ solution was used as an electrolyte in linear sweep voltammetry (LSV) measurements with a scan rate of $5 \mathrm{mV} \mathrm{s}^{-1}$; c) corresponding Tafel plots produced from LSV measurements of different porous hollow fiber cathodes and Pt/C. The curves in the low current density region were considered for Tafel plots. d) Nyquist plots of electrochemical impedance spectroscopy (EIS) spectra (in $\mathrm{H}_{2} \mathrm{SO}_{4}$ solution at $0 \mathrm{~V}$ ) of $\mathrm{Pt} / \mathrm{C}$ and porous hollow fiber cathodes developed at different cycles of ALD Pt catalyst. 


\section{WILEY-VCH}

The results of ALD cycles below 150 were not presented because the electro-analysis of the porous hollow fiber cathodes was only supported when the Pt deposition was $\geq 150$ cycles, perhaps due to poor electronic conductivity associated to the discontinuity of the Pt layer obtained with less cycles on a non-conductive polymer surface. ${ }^{[13]}$ As the number of ALD cycles increased, the deposited Pt amount (as per inductively coupled plasma mass spectrometry measurement) also increased, while the water permeation decreased (i.e. higher TMP) (Figure 1a). The higher TMP needed to maintain the same water flux after the ALD deposition was due to the reduction in pore size. ${ }^{[16]}$ The TMP of the unmodified POD hollow fiber membrane was 0.0003 bar.

The electrochemical performance of the porous hollow fiber cathodes prepared at different numbers of ALD Pt cycles toward the HER was evaluated by linear sweep voltammetry (LSV) (Figure 1b). As a reference point, we also performed measurements using benchmark Pt-coated carbon $\left(\mathrm{Pt} / \mathrm{C}\right.$ ) cathode (Pt concentration of $0.5 \mathrm{mg} \mathrm{cm}^{-2}$ ) having high HER catalytic performance (onset overpotential of $0.01 \mathrm{~V}$ ). From LSV analysis, we inferred a strong correlation between the number of ALD Pt cycles and HER activity. Although the onset overpotential for HER was more or less the same (i.e. between 0.01 to $0.03 \mathrm{~V}$ vs reversible hydrogen electrode (RHE)) for the porous hollow fiber cathodes prepared at different numbers of ALD cycles (Figure 1b), the overpotentials for generating high HER current densities $\left(10 \mathrm{~mA} \mathrm{~cm}{ }^{-2}\right)$ varied with the number of ALD Pt cycles. The overpotentials to produce a cathodic current density of $\sim 10 \mathrm{~mA} \mathrm{~cm}{ }^{-2}$ decreased with the increase in the number of ALD Pt cycles (Figure 1b), and hence Pt loading (Figure 1a). This increase in Pt loading enhanced the connectivity between the Pt particles and increased the number of active catalytic sites for improving the HER activity ${ }^{[17]}$. Moreover, the overpotential of the porous hollow fiber cathode fabricated with 250 cycles of ALD $(0.19 \mathrm{~V} v s$. RHE) for generating high HER current density of $10 \mathrm{~mA} \mathrm{~cm}^{-2}$ was lower than the overpotential of the benchmark cathode (Pt/C) (0.23 V vs. RHE) despite $\sim 53 \%$ lower Pt loading $(0.233 \mathrm{mg}$ $\mathrm{cm}^{-2}$ ). This clearly demonstrates the advantage of ALD approach by allowing a more efficient 


\section{WILEY-VCH}

utilization of $\mathrm{Pt}$ (thus minimizing amount of $\mathrm{Pt}$ ) even on three-dimensional porous hollow fibers through a combination of highly uniform coating and precise control of size, density and shape of deposited Pt on the surface. ${ }^{[18]}$

Low Tafel slopes and high exchange-current density $\left(j_{o}\right)$ are an indication of high performance HER electrocatalyst activity. ${ }^{[19]}$ The influence of the number of ALD Pt cycles on the HER activity of the porous hollow fiber cathodes was further confirmed by Tafel plots (Figure 1c). The cathodes obtained with 150 cycles of ALD Pt catalyst had a significantly higher Tafel slope $\left(160 \mathrm{mV} \mathrm{dec}^{-1}\right)$ compared to the ones prepared with ALD cycles $\geq 200(50-68 \mathrm{mV}$ $\left.\operatorname{dec}^{-1}\right)$. The high Tafel slope at 150 cycles of ALD Pt could be due to lower connectivity between the Pt particles and lower number of active catalytic sites. Notably, the Tafel slopes for the porous hollow fiber cathodes prepared with ALD cycles $\geq 200$ were comparable to that of the benchmark cathode (Pt/C) $\left(51 \mathrm{mV} \mathrm{dec}^{-1}\right)$ (Figure 1c) despite lower Pt loadings (0.207 to 0.325 $\mathrm{mg} \mathrm{Pt} \mathrm{cm}^{-2}$ ). Usually, low Tafel slopes are desirable for practical applications since it allows a faster increase of the HER current density with increasing overpotentials. ${ }^{[20]}$ The Tafel slope is often utilized to indicate the electrochemical HER mechanism. The Tafel slope should be 120, 40 or $30 \mathrm{mV} \mathrm{dec}^{-1}$ if Volmer (or discharge), Heyrovsky (or electrochemical desorption), or Tafel (or recombination) reaction is the rate-limiting step of the HER. ${ }^{[17,21]}$ The observed Tafel slopes for the porous hollow fiber cathodes prepared with ALD cycles $\geq 200$ and the benchmark $\mathrm{Pt} / \mathrm{C}$ cathode suggest that electrochemical desorption is the rate-limiting step and the VolmerHeyrovsky mechanism for HER.

The $j_{o}$ is a key parameter of the interfacial reaction kinetics. ${ }^{[13]}$ The $j_{o}$ for the various porous hollow fiber cathodes developed at 150, 200, 250, 300 and 400 cycles of ALD and benchmark $\mathrm{Pt} / \mathrm{C}$ cathode were $0.073,0.086,0.160,0.220,0.450$ and $0.110 \mathrm{~mA} \mathrm{~cm}^{-2}$, respectively. These results suggest that higher $j_{o}$ (i.e. faster interfacial kinetics) were obtained at higher numbers of ALD Pt cycles, perhaps due to the increase in the number of active catalytic sites per surface 


\section{WILEY-VCH}

area. Chao et al. (2012) observed higher $j_{o}$ for Pt electrodes, prepared by ALD, that had higher reaction sites per unit area. ${ }^{[13]}$

The enhanced HER performance of the porous hollow fiber cathodes with increase in number of cycles of ALD Pt catalyst was attributed to the decrease in the electron transfer resistance. This was confirmed by electrochemical impedance spectroscopy (EIS). The Nyquist plots (Figure 1d) of the EIS spectra of the porous hollow fiber cathodes revealed that the ohmic resistance decreased from 120 to $13 \Omega$ with increase in the number of ALD cycles. Increasing the number of ALD cycles increased the connectivity between Pt particles, thus reducing the effect of ohmic losses, in particular losses arising from the flow of electrons through the cathode.

The porous hollow fiber cathodes fabricated with 200 cycles of ALD Pt catalyst (Figure 2) were selected for testing in EMBR experiments, due to, lower Pt loading $(0.207 \pm 0.001 \mathrm{mg} \mathrm{Pt}$ $\mathrm{cm}^{-2}$ ) compared to ALD cycles $\geq 250$, lower ohmic resistance for achieving high electronic conductivity and HER current densities compared to 150 ALD cycles and exchange-current density and Tafel slope values comparable to that of the benchmark cathode (Pt/C) (Figure 1a,c,d). From here onwards, all discussed experiments were performed with porous hollow fiber cathodes fabricated with 200 cycles of ALD Pt catalyst. The Transmission Electron Microscopy (TEM) images confirm the formation of Pt thin film $(26 \pm 5 \mathrm{~nm})$ layering on the outer surface of the POD hollow fiber membrane (Figure 2b-c). The thickness of the Pt layer was sufficient to achieve good electronic conductivity and electrochemical activity (Figure 1b-d). High resolution TEM image of the Pt film confirms that the catalyst layer on the POD hollow fiber membrane surface is assembled by nano-sized Pt particles $(\sim 5 \mathrm{~nm})$ (Figure $2 \mathrm{~d})$. This nanostructured Pt catalyst on the surface of POD hollow fiber membrane by ALD technique is advantageous as it increases the number of active catalytic sites per mass of catalyst, thus resulting in better Pt utilization. ${ }^{[18]} \mathrm{Pt}$ distribution on the surface of the POD hollow fiber membrane was further confirmed using Energy-dispersive X-ray spectroscopy (EDS) analysis (Figure 2e,f). According to the line scan measurements and elemental mapping, more Pt was 


\section{WILEY-VCH}

deposited on the pore borders and the pores were clearly not blocked. Also, the porosity (determined by liquid pore filling) of unmodified POD hollow fiber membranes and porous hollow fiber cathodes fabricated with 200 cycles of ALD Pt was $81.8 \pm 0.6 \%$ and $80.1 \pm 0.3 \%$, respectively. These results confirm that the ALD technique has the ability to grow catalyst layers on porous hollow fiber membranes with minimal pore filling/blockage during Pt coating.

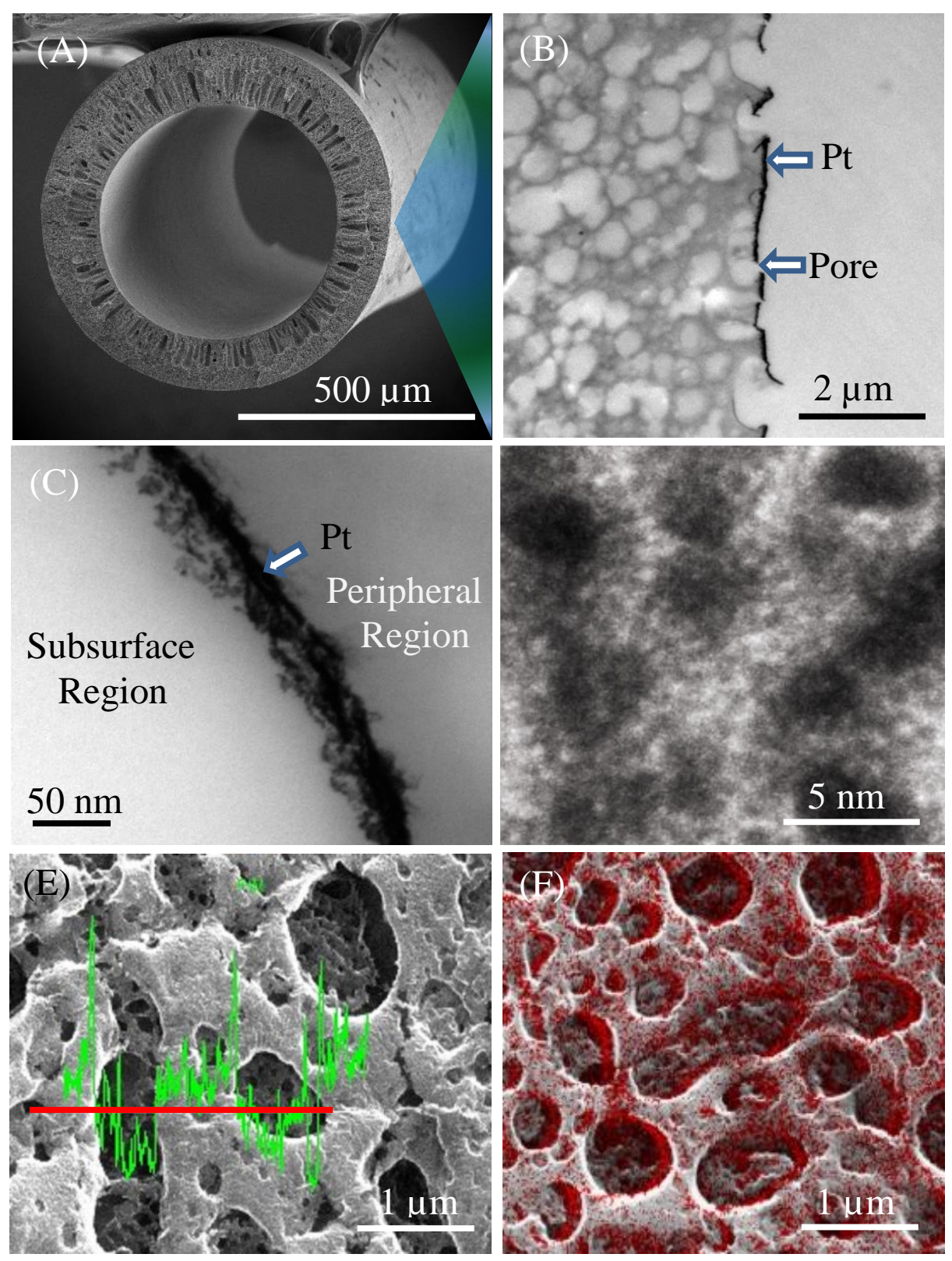

Figure 2. Morphological characterization of the porous hollow fiber cathode fabricated with 200 cycles of ALD Pt catalyst. a) Scanning electron microscope image of cross-section of virgin porous hollow fiber cathode; b) Transmission electron microscope (TEM) image (prepared by microtome) showing close-up view of the porous hollow fiber cathode outer edge cross-section; 


\section{WILEY-VCH}

c) TEM image showing the morphology of Pt layer; d) TEM image showing close-up view of the Pt layer; e) energy-dispersive X-ray spectroscopy (EDS) line-scan; and f) EDS mapping. The red color on the image represent the Pt localization on the surface of the porous hollow fiber cathode.

Porosimetry analysis of the pristine POD hollow fiber membranes and the porous hollow fiber cathodes fabricated with 200 cycles of ALD Pt catalyst revealed that ALD of Pt finely tuned the pore size on the membranes surface (Figure S2), which is advantageous for improving the separation performance in water systems. The POD hollow fiber membranes had a multimodal pore size distribution with diameters in the following ranges: $300-320 \mathrm{~nm}, 370-410$ $\mathrm{nm}$ and 530-600 nm. After the Pt deposition the pore size distribution was reduced with peaks at $170 \mathrm{~nm}$ and $210 \mathrm{~nm}$. The resulting tuned pore profile with decreased pore diameter could effectively block the passage of bacteria, as a consequence permeate quality was greatly enhanced, which will be detailed later. These results highlight the advantage of ALD as an effective technique for tailoring the pore microstructure of the porous hollow fiber cathodes through gearing conformal layering of catalyst with a controllable thickness. ${ }^{[9,11]}$

In order to evaluate the robustness and efficiency of the developed porous hollow fiber cathodes for water and energy recovery, experiments were conducted in an anaerobic EMBR (Figure 3) operated with a working electrode (anode) applied potential of $-0.1 \mathrm{~V}$ vs $\mathrm{Ag} / \mathrm{AgCl}$. Experiments were conducted using acetate solution or sewage (wastewater) as the source of electron donor for electroactive bacteria $(\mathrm{EAB})$ as well as electrolyte. The EAB on the surface of carbon fiber brush anode (Figure S3) transport the electrons generated during the oxidation of acetate or organics in wastewater to the anode. The electrons and protons that are generated during oxidation at the anode ultimately are reduced to $\mathrm{H}_{2}$ on the surface of the porous hollow fiber cathode. At the end of each experimental batch cycle, the treated medium was filtered through the cathode at a permeate flux of $12 \mathrm{~L} \mathrm{~m}^{-2} \mathrm{~h}^{-1}$. 


\section{WILEY-VCH}

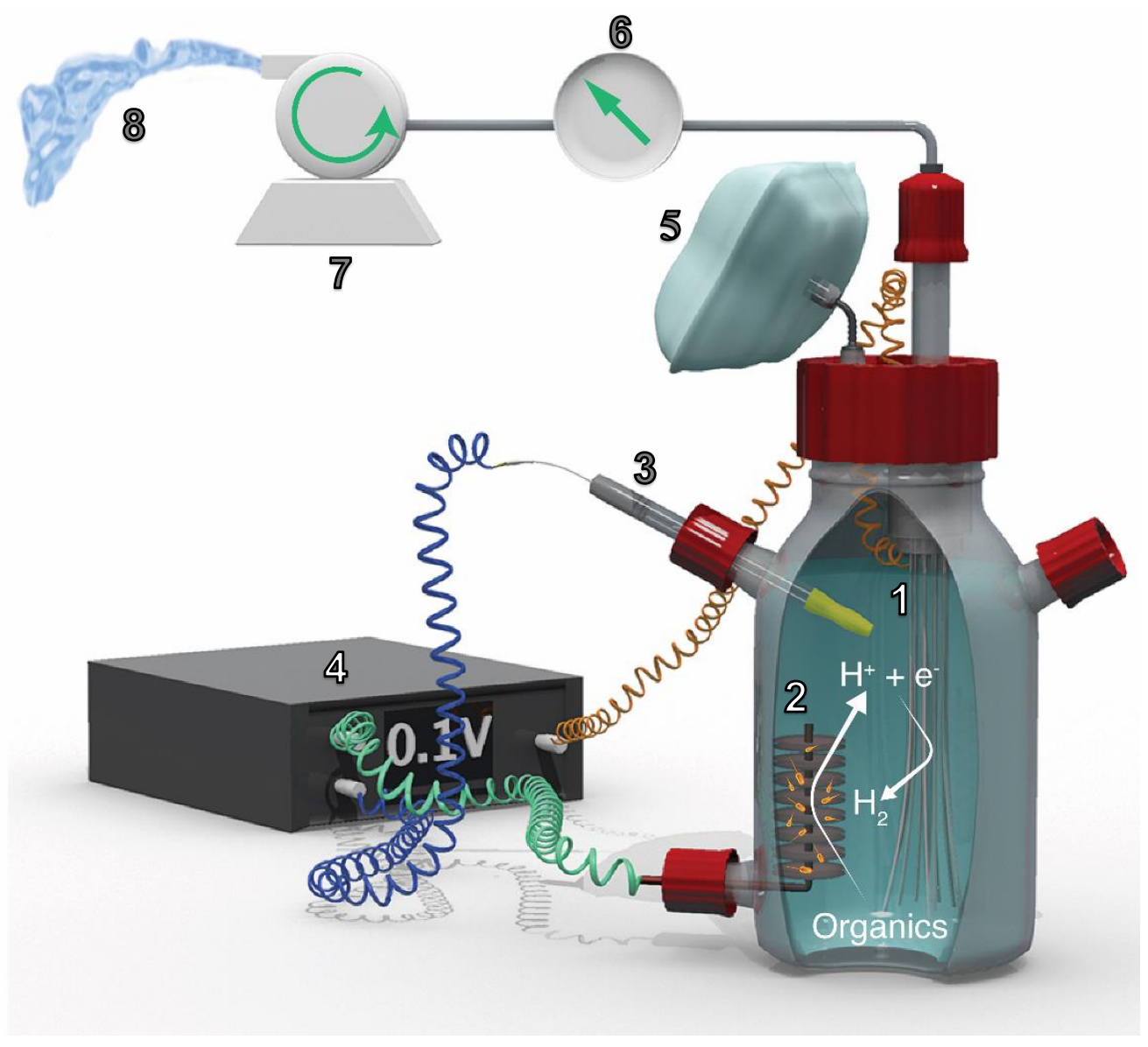

Figure 3. Schematic representation of the electrochemical membrane bioreactor (EMBR). 1) porous hollow fiber cathode bundle (counter electrode); 2) Carbon fiber brush anode (working electrode); 3) $\mathrm{Ag} / \mathrm{AgCl}$ refernce electrode; 4) potentiostat; 5) gas collection bag; 6) pressure sensor; 7) perstaltic pump; 8) permeate.

Figure 4 summarizes the results of peak current generation and gas evolution under $-0.1 \mathrm{~V}$ vs $\mathrm{Ag} / \mathrm{AgCl}$ applied potential, and TMP variation with respect to time during the fed-batch operation of the EMBR. During the reactor startup using acetate solution as electron donor, operating with $400 \mathrm{mg} \mathrm{L}^{-1}$ Chemical Oxygen Demand (COD) and solution conductivity 6.96 $\mathrm{mS} \mathrm{cm}{ }^{-1}$, the current gradually increased with time up to $400 \mathrm{~h}$ of fed-batch operation (Figure 4a). This can be attributed to the acclimatization period needed for the formation of a stable anodic biofilm (biocatalyst), which supports the oxidation reaction on the anode. 

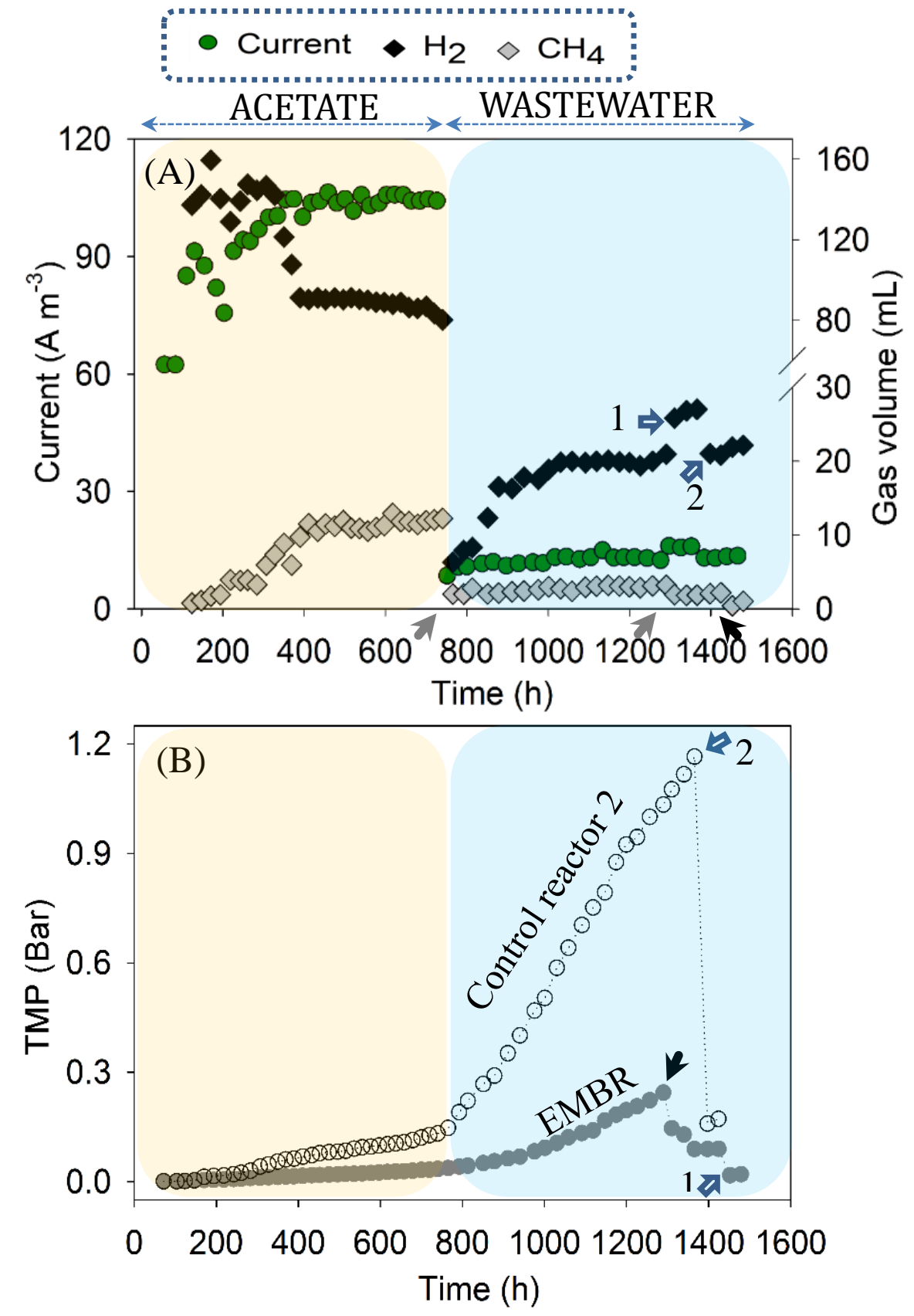

Figure 4. a) Peak volumetric current density and volumes of gas produced from the EMBR over the course of the experiment with acetate solution or wastewater as fuel source. Porous hollow fiber cathodes prepared at 200 cycles of ALD Pt catalyst $\left(0.2 \mathrm{mg} \mathrm{Pt} \mathrm{cm}{ }^{-2}\right)$ having an active surface area of $\sim 10 \mathrm{~cm}^{2}$ were used as the cathode and filter material. Reactors were operated in fed-batch mode, and each data point in the plot represents the respective performance of individual batch cycles. The duration of each batch cycle was $22.8 \pm 2.7 \mathrm{~h}$ with acetate and $30 \pm 2.6 \mathrm{~h}$ with wastewater. Glucose was used to adjust the wastewater (feed) chemical oxygen demand concentration to $\sim 450 \mathrm{mg} \mathrm{L}^{-1}$ throughout the study. Gray color 


\section{WILEY-VCH}

arrows show the points when the biofouled porous hollow fiber cathodes were sampled for confocal laser scanning microscopy and scanning electron microscope analysis. Un-filled arrows represent the time points at which the applied potential of working electrode (anode) was ramped to $+0.1 \mathrm{~V}$ from $-0.1 \mathrm{~V}$ vs $\mathrm{Ag} / \mathrm{AgCl}$ (arrow 1) and switched back to $-0.1 \mathrm{~V}$ (arrow 2). Black arrow represents the time where the biofouled porous hollow fiber cathodes were subjected to physical/chemical cleaning; b) variations in TMP at a fixed flux $\left(12 \mathrm{~L} \mathrm{~m}^{-2} \mathrm{~h}^{-1}\right)$ in the EMBR (filled circles) and control reactor 2 (Un-filled circles) over the course of the experiment with acetate solution or wastewater as fuel source. Black arrow represents the time point at which the applied potential of working electrode was ramped to $+0.1 \mathrm{~V}$ from $-0.1 \mathrm{~V}$ vs $\mathrm{Ag} / \mathrm{AgCl}$. Un-filled arrow 1 represents the time when the biofouled porous hollow fiber membranes in the EMBR was subjected to physical/chemical cleaning. Un-filled arrow 2 represents the time (at $1374 \mathrm{~h}$ ) when the control reactor 2 (initially operated under open circuit mode) was operated under electrolysis mode at an applied voltage of $2 \mathrm{~V}$ using a power source.

The Coulombic efficiency (CE) in the EMBR was $>100 \%$ during the first $327 \mathrm{~h}$ of operation with acetate (Figure S4). Coulombic efficiencies of $>100 \%$ have been reported previously due to hydrogen recycling by EAB using hydrogen as the electron donor. ${ }^{[22]}$ However, when significant methanogenesis was observed in the system after $350 \mathrm{~h}$ of operation (Figure S4) the CE was reduced to $96.7 \pm 0.3 \%$, likely due to efficient conversion of hydrogen to methane mainly by hydrogenotrophic methanogens in the cathode biofilm. Hydrogenotrophic methanogensis was supported by i) the absence of methane gas when the EMBR was operated under open circuit mode (i.e. no HER) at the end of the experiment with acetate medium; and ii) absence of methane in the headspace after the biofouled porous hollow fiber cathodes were subjected to physical/chemical cleaning (at 1420 h) (Figure 4a). In contrast, the EMBR performance (current density and $\mathrm{H}_{2}$ evolution) remained stable after cleaning. Also, our previous EMBR studies showed that methane generation was mainly via hydrogenotrophic methanogens in the cathode biofilm. ${ }^{[4,23]}$ In addition, most microbial electrolysis cell studies 


\section{WILEY-VCH}

showed that hydrogenotrophic methanogens are primarily responsible for methane generation because acetate-oxidizing EAB could outcompete acetoclastic methanogens for acetate due to kinetic benefits. ${ }^{[24,25]}$ In contrast, hydrogenotrophic methanogens could outcompete EAB for $\mathrm{H}_{2}$ because of their thermodynamic and kinetic benefits. ${ }^{[24,26]}$

The cathodic recovery of hydrogen $\left(r_{\mathrm{Cat}} \mathrm{H}\right)$ was the highest $(>95 \%)$ during the first $327 \mathrm{~h}$ of operation and then gradually dropped to reach $61 \%$ at $410 \mathrm{~h}$ and remained steady throughout the experimental period with acetate. In contrast, the cathodic recovery of methane $\left(r_{\mathrm{Cat}} \mathrm{CH} 4\right)$ was very low during the first $200 \mathrm{~h}$ of operation and then gradually increased to reach $34 \%$ at $430 \mathrm{~h}$ and remained steady throughout the experimental period with acetate.

After observing stable reactor operation (Current: $104 \pm 0.3 \mathrm{~A} \mathrm{~m}^{-3}$ and Coulombic efficiency, CE: $96.7 \pm 0.3 \%$ ) over the period from $400 \mathrm{~h}$ to $740 \mathrm{~h}$ of EMBR operation with acetate solution (Figure 4a), the reactors were fed with real domestic wastewater $\left(450 \pm 28 \mathrm{mg} \mathrm{L}^{-1} \mathrm{COD} ; 740\right.$ to $1465 \mathrm{~h}$ ) in order to test the efficacy of the porous hollow fiber cathodes for i) energy recovery, ii) biofouling mitigation, and iii) high quality water (permeate) recovery. The EMBR produced an average current density of $12.9 \pm 0.7 \mathrm{~A} \mathrm{~m}^{-3}$ after switching the fuel source from acetate to domestic wastewater. The hydrogen evolution rate at stable current generation in the EMBR was higher with acetate $\left(0.34 \pm 0.01 \mathrm{~m}^{3} \mathrm{~m}^{-3} \mathrm{~d}^{-1}\right)$ than with wastewater $\left(0.074 \pm 0.009 \mathrm{~m}^{3} \mathrm{~m}^{-3} \mathrm{~d}^{-}\right.$ ${ }^{1}$ ) (Figure 4a). Although the operating conditions were the same with both fuel sources, the lower electrochemical performance with real wastewater was due to the complexity of the organics (which serve as the fuel source) and lower wastewater conductivity $\left(1.43 \mathrm{mS} \mathrm{cm} \mathrm{cm}^{-1}\right)$, which reduced the Coulombic efficiency $(\mathrm{CE}, 22.3 \pm 1.6 \%)$ by minimizing the anodic biocatalysis. ${ }^{[27]}$ The measured cathode potentials ( -0.7 V vs RHE) during the EMBR operation with wastewater (Figure S5) was much lower than the overpotential required for attaining maximum HER current densities (i.e. $10 \mathrm{~mA} \mathrm{~cm}^{-2}$ ) with porous hollow fiber cathodes fabricated at 200 cycles of ALD (i.e. $-0.32 \mathrm{~V} v s$ RHE) (Figure 1b). This suggests that the measured porous hollow fiber cathode potential during the reactor operation well support the HER activity, and 


\section{WILEY-VCH}

thus supports the anodic reaction under the operational conditions tested in this experiment.

The EMBR performance (current density and $\mathrm{H}_{2}$ evolution) remained stable after chemical/physical cleaning (at $1420 \mathrm{~h}$ ) of the porous hollow fiber cathodes to remove biological foulants (Figure 4a). This suggests that the adopted membrane cleaning procedure did not affect the electrocatalytic properties of the fibers, demonstrating robustness of the ALD Pt coating. It is also noteworthy that the EMBR was operated for $1500 \mathrm{~h}$ ( 62 days) with negligible loss of current density (Figure 4a), suggesting high stability of the electrocatalytic component of the porous hollow fiber cathodes fabricated by the ALD process.

A similar trend in electrochemical performance in terms of current density and CE (@ stable current generation; acetate: $115 \pm 8.7 \mathrm{~A} \mathrm{~m}^{-3}$, CE 98.6 $\pm 4.1 \%$ and wastewater: $13.8 \pm 0.9$ A m ${ }^{-3}, \mathrm{CE}: 24.4 \pm 1.7 \%$ ) and $\mathrm{H}_{2}$ evolution (acetate: $0.37 \pm 0.01 \mathrm{~m}^{3} \mathrm{~m}^{-3} \mathrm{~d}^{-1}$ and wastewater: 0.09 $\pm 0.01 \mathrm{~m}^{3} \mathrm{~m}^{-3} \mathrm{~d}^{-1}$ ) was observed in the control reactor 1 (Figure S6). The control reactor 1 was constructed similarly to the EMBR and was operated in parallel under the same conditions as the EMBR (-0.1 V Ag/AgCl applied potential and cathode specific surface area $\left.3.27 \mathrm{~m}^{2} \mathrm{~m}^{-3}\right)$, but using benchmark Pt-carbon cloth cathode having the same active surface area $\left(\sim 10 \mathrm{~cm}^{2}\right)$ as the porous hollow fiber cathodes used in the EMBR. These results suggest that the differences in EMBR performance with respect to the fuel type are not associated with the porous hollow fiber cathodes architecture or catalyst activity since similar behavior was observed in control reactor 1 when operated with acetate or wastewater. Despite $60 \%$ lower mass of Pt per unit surface area of the cathode, the EMBR achieved similar electrochemical performance as attained with the control reactor 1 , which had a carbon cloth cathode coated with $0.5 \mathrm{mg} \mathrm{Pt} \mathrm{cm}-$

${ }^{2}$. The precise control of Pt loading, size and density by the ALD technique on the porous hollow fiber membranes resulted in an enhanced electrocatalytic HER activity at lower mass of Pt. Therefore, ALD is a promising technique to fabricate electrodes with high electrocatalytic activity by creating catalyst structures with high surface areas, while minimizing the total mass (and therefore cost) of the catalyst. ${ }^{[12,28]}$ 


\section{WILEY-VCH}

Membrane fouling contributes significantly to both operation and capital costs in membrane bioreactors that arise from the need to clean or replace membranes. ${ }^{[29]}$ The increase in TMP (a measure of membrane fouling propensity) in the EMBR with time (Figure 4b) was minor with acetate solution $(0.035 \pm 0.01$ bar over $727 \mathrm{~h}$ operation $)$. When wastewater was introduced in the EMBR a faster increase in TMP was recorded, rising from $0.035 \pm 0.01$ to $0.24 \pm 0.02$ bar after 549 hours. However, this value was much lower than that observed with the control reactor 2 (EMBR operated under the same conditions but in open circuit mode) in the same period of time, for which the pressure needed to be increased to 1 bar or higher to maintain the water flux of $12 \mathrm{~L} \mathrm{~m}^{-2} \mathrm{~h}^{-1}$. Also, the increase in TMP in the EMBR was 3 times lower than control reactor 2 (Figure 4b). Confocal laser scanning microscopy (Figure 5) and SEM (Figure S7) images of biofouled porous hollow fiber cathodes collected from the EMBR and control reactor 2 at two time intervals (740 h and 1374 h; Figure $4 a$ ) confirmed that biofouling increased with time, and it became more aggressive when the reactor operation was switched to wastewater instead of acetate solution. A substantial reduction in TMP $(0.24 \pm 0.02$ to $0.09 \pm 0.04$ bar) was noticed (after $1276 \mathrm{~h}$, Figure $4 \mathrm{~b}$ ) when the hydrogen evolution rate in the EMBR was increased by switching the applied potential from $-0.1 \mathrm{~V}$ to $+0.1 \mathrm{~V} v s \mathrm{Ag} / \mathrm{AgCl}$ (after 1276 h, Figure 4a). This suggests that the biofilm developed on the surface of the porous hollow fiber cathode was disturbed by the enhanced $\mathrm{H}_{2}$ evolution rates $\left(0.1 \pm 0.02 \mathrm{~m}^{3} \mathrm{~m}^{-3} \mathrm{~d}\right)$, resulting from the potential switch. It is known that $\mathrm{H}_{2}$ evolution rates can be enhanced in microbial electrolysis cells by increasing the applied potential of working electrode (i.e. anode), thus intermittent switching of the anode operating potentials can be used as a strategy to mitigate biofouling in the EMBR system. ${ }^{[30]}$ 
WILEY-VCH

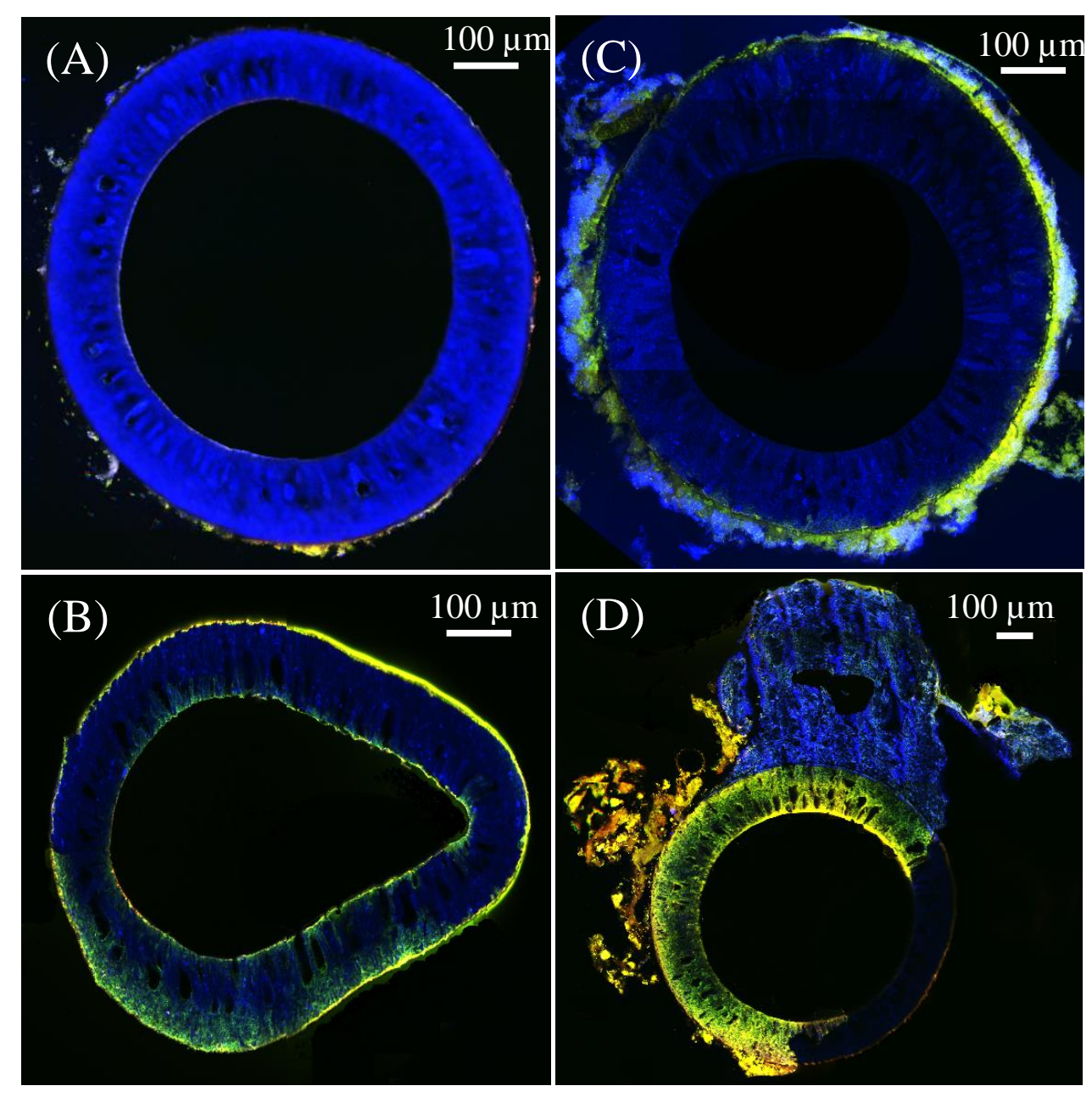

Figure 5. Confocal laser scanning microscope images of biofouled porous hollow fiber cathodes. Images A (EMBR) and B (Control reactor 2) represent a cross section of the biofouled porous hollow fiber cathodes collected after $740 \mathrm{~h}$ of fed-batch operation using acetate solution as the fuel source. Images C (EMBR) and D (Control reactor 2) represent the biofouled porous hollow fiber cathodes collected at $1374 \mathrm{~h}$ (i.e. after $\sim 630 \mathrm{~h}$ of fed-batch operation using wastewater as the fuel source). Bacterial cells (green), proteins (blue) and $\alpha$ - and $\beta$ Polysaccharides (red and yellow) were stained with their corresponding dyes (Table S1, Supporting information).

To further confirm the effect of hydrogen evolution rate in the in-situ cleaning of the membranes, the control reactor 2 (initially operated under open circuit mode) was then operated under electrolysis mode (unfilled arrow 2 in Figure 4b) at an applied voltage of $2 \mathrm{~V}$, using a power source. A platinum mesh electrode $(2 \mathrm{~cm} \times 3 \mathrm{~cm})$ as anode (replacing the fiber brush 


\section{WILEY-VCH}

anode) for water electrolysis was positioned parallel to the biofouled (1374 h aged) porous hollow fiber cathodes in the control reactor 2 and the hydrogen evolution reaction was triggered. A drastic drop of TMP ( $86 \%$ reduction) was observed, after 30 min of electrolysis (Figure 4b) with a hydrogen evolution rate of $1.45 \mathrm{~m}^{3} \mathrm{~m}^{-3} \mathrm{~d}^{-1}$. Also, confocal laser scanning microscopy showed a noticeable difference in the biofilm coverage for the porous hollow fiber cathode operated under open circuit mode and following operation under electrolysis mode (Figure S8). These results clearly demonstrate the role of in-situ cleaning by the hydrogen bubble formation at the membrane surface in biofouling mitigation in the EMBR system. Although measurements were not carried out in this study, we cannot rule out the possibility that other physico-chemical factors associated with HER activity, such as the localized high $\mathrm{pH}$ at the cathode surface due to reduction of $\mathrm{H}^{+}$and the negative potential and electrical charge of the cathode (electrostatic repulsive force between the negatively charged cathode and similarly charged foulants), might have contributed for biofouling mitigation in the EMBR. ${ }^{[31]}$

The performance of the EMBR was also evaluated in terms of wastewater treatment efficiency (i.e. COD removal) and permeate quality. Significant removal of COD was achieved with slight differences in removal efficiency observed with respect to the type of fuel tested (acetate $95 \pm 2.8 \%$ and wastewater $85 \pm 1.4 \%)$. The $\mathrm{pH}$ of the acetate feed $(7.25 \pm 0.21)$ was not significantly different from wastewater $(7.65 \pm 0.21)$, whereas the effluent $\mathrm{pH}$ with wastewater $(8.6 \pm 0.3)$ was higher than acetate $(7.37 \pm 0.17)$. No buffer medium was added to wastewater, whereas a buffer medium was added to the acetate solution. The higher $\mathrm{pH}$ in the non-buffered wastewater was due to consumption of protons at the cathode for HER. Similar COD removals were observed in the control reactor 1 (acetate $97 \pm 1.4 \%$ and sewage $83 \pm$ 2.3\%) operated with Pt-carbon cloth cathode. The custom-built porous hollow fiber cathodes well supported the anodic reaction (i.e. COD removal and CE generation) even with $60 \%$ lower mass of Pt per surface area. High quality water (permeate) with turbidity $<0.1$ nephelometric turbidity units was recovered from the EMBR (Figure S9). This result confirms the filtration 


\section{WILEY-VCH}

efficiency of the tested porous hollow fiber cathode architecture for reclamation of treated effluents.

In conclusion, we have developed an electro-catalytic and porous polymeric hollow fiber with dual function (recovery of clean water and energy) by combining the fabrication of thermally stable polymeric hollow fiber membrane by phase inversion followed by deposition of a thin film of nano-Pt at $180^{\circ} \mathrm{C}$ on the surface of the porous polymeric hollow fibers by ALD. The catalytic activity of the developed porous hollow fiber cathode for HER was observed for as little as 150 ALD cycles. The electro-catalytic and filtration efficacy of the porous hollow fiber cathodes prepared with 200 cycles of ALD Pt catalyst were tested in EMBR experiments using acetate or sewage as fuel source. Despite significantly lower Pt loading, the electrochemical performance of the porous hollow fiber cathode was comparable to the benchmark Pt-carbon cloth cathode. The ALD technique facilitated the uniform coverage of the porous hollow fibers with low Pt loading, which minimizes the costs associated with the use of noble-metal catalysts. Importantly, the idea of the porous hollow fiber cathode is not limited to Pt but lends itself to other low cost materials and for further improvement and optimization, to reduce fabrication costs even further. By using ALD method, we were able to fine tune the pore size of the porous hollow fibers resulting in high quality water enabling reclamation of treated effluent. The superior performance of the porous hollow fiber cathode for membrane fouling mitigation was attributed to the in-situ cleaning by hydrogen bubble formation and other factors associated with HER activity. We believe that these polymeric hollow fibers that are electrocatalytic and porous have a broader application landscape. For example, they can be used as a microfiltration membrane in membrane bioreactors for wastewater treatment and reuse, and if integrated with an external electric field they have the potential to solve the biofouling issue in membrane bioreactors. Also, the 3D porous hollow fiber architecture of the electrodes makes them ideal for use as gas diffusion electrodes to stimulate electrochemical reactions such as the 


\section{WILEY-VCH}

conversion of $\mathrm{CO}_{2}$ to value added products through microbial electrosynthesis or

electrochemical conversion. ${ }^{[5]}$

\section{Supporting Information}

Supporting Information is available from the Wiley Online Library or from the author.

\section{Acknowledgements}

K.K. and N.M.S.B contributed equally to this work. This work was supported by Center Competitive Funding Program (FCC/1/1971-05-01) from King Abdullah University of Science and Technology. We thank Mr. Hyun ho Hwang (Heno) at the Office of Academic Writing Services at KAUST for generating the ToC figure in this manuscript.

[1] R. Connor, S. Grego, M. Miletto, O. Unver, in Water and Energy, Vol. 1 (Ed: W. U. N. W. W. A. Programme)), UNESCO, Paris 2014.

[2] L. Malaeb, K. P. Katuri, B. E. Logan, H. Maab, S. P. Nunes, P. E. Saikaly, Environ. Sci. Technol. 2013, 47, 11821; Y.-K. Wang, G.-P. Sheng, W.-W. Li, Y.-X. Huang, Y.-Y. Yu, R. J. Zeng, H.-Q. Yu, Environ. Sci. Technol. 2011, 45, 9256; P. Zhang, Y. Qu, J. Liu, Y. Feng, RSC Adv. 2016, 6, 4587; K. Zuo, S. Liang, P. Liang, X. Zhou, D. Sun, X. Zhang, X. Huang, Bioresour. Technol. 2015, 185, 426.

[3] H. Q. Hussein, P. de Wit, E. J. Kappert, N. E. Benes, ACS Sustainable Chem. Eng. 2015, 3, 3454.

[4] K. P. Katuri, C. M. Werner, R. J. Jimenez-Sandoval, W. Chen, S. Jeon, B. E. Logan, Z. Lai, G. L. Amy, P. E. Saikaly, Environ. Sci. Technol. 2014, 48, 12833.

[5] R. Kas, K. K. Hummadi, R. Kortlever, P. de Wit, A. Milbrat, M. W. J. LuitenOlieman, N. E. Benes, M. T. M. Koper, G. Mul, Nat. Commun. 2016, 7.

[6] N. C. Mat, Y. Lou, G. G. Lipscomb, Curr. Opin. Chem. Eng. 2014, 4, 18.

[7] F. Ahmed, B. S. Lalia, V. Kochkodan, N. Hilal, R. Hashaikeh, Desalination. 2016, $391,1$.

[8] O. K. Duaij, A. Alghamdi, Z. Y. Al-Saigh, J. Chromatogr. A. 2013, 1291, 137.

[9] N. P. Dasgupta, C. Liu, S. Andrews, F. B. Prinz, P. Yang, J. Am. Chem Soc. 2013, $135,12932$.

[10] R. W. Johnson, A. Hultqvist, S. F. Bent, Mater. Today. 2014, 17, 236.

[11] A. M. Schwartzberg, D. Olynick, Adv. Mater. 2015, 27, 5778.

[12] J. W. Elam, N. P. Dasgupta, F. B. Prinz, MRS Bull. 2011, 36, 899.

[13] C.-C. Chao, M. Motoyama, F. B. Prinz, Adv. Energy. Mater. 2012, $2,651$.

[14] K.-V. P. Suzana Pereira Nunes, WILEY-VCH Verlag GmbH \& Co. KGaA. 2006.

[15] K. D.-C. Delphine Longrie, Davy Deduytsche, Sven Van den Berghe, Kris Driesen and Christophe Detavernier, ECS J. Solid State Sci.Technol. 2012, 1.

[16] F. Li, L. Li, X. Liao, Y. Wang, J. Membrane. Sci. 2011, 385-386, 1.

[17] Y. Li, H. Wang, L. Xie, Y. Liang, G. Hong, H. Dai, J. Am. Chem Soc. 2011, 133, 7296.

[18] A. Kloke, F. von Stetten, R. Zengerle, S. Kerzenmacher, Adv. Mater. 2011, 23, 4976.

[19] Y. Zheng, Y. Jiao, Y. Zhu, L. H. Li, Y. Han, Y. Chen, A. Du, M. Jaroniec, S. Z. Qiao, Nat. Commun. 2014, 5.

[20] Y. Hou, B. Zhang, Z. Wen, S. Cui, X. Guo, Z. He, J. Chen, J. Mater. Chem. A. 2014, 2,13795 . 


\section{WILEY-VCH}

[21] W. Cui, Q. Liu, N. Cheng, A. M. Asiri, X. Sun, Chem. Commun. 2014, 50, 9340; W.F. Chen, J. T. Muckerman, E. Fujita, Chem. Commun. 2013, 49, 8896.

[22] L. Lu, D. Xing, T. Xie, N. Ren, B. E. Logan, Biosens. Bioelectron. 2010, 25, 2690; J. M. Pisciotta, Z. Zaybak, D. F. Call, J. Y. Nam, B. E. Logan, Appl. Environ. Microbiol. 2012, 78,5212 .

[23] C. M. Werner, K. P. Katuri, A. R. Hari, W. Chen, Z. Lai, B. E. Logan, G. L. Amy, P. E. Saikaly, Environ. Sci. Technol. 2016, 50, 4439.

[24] Y. Gao, H. Ryu, J. W. Santo Domingo, H. S. Lee, Bioresour. Technol. 2014, 153, 245.

[25] S. Jung, J. M. Regan, Appl. Environ. Microbiol. 2011, 77, 564; L. Lu, D. Xing, N.

Ren, Environ. Sci. Technol. 2012, 46, 6874; P. Parameswaran, C. I. Torres, H. S. Lee, R.

Krajmalnik-Brown, B. E. Rittmann, Biotechnol. and Bioeng. 2009, 103, 513.

[26] H. S. Lee, C. I. Torres, P. Parameswaran, B. E. Rittmann, Environ. Sci. Technol. 2009, $43,7971$.

[27] E. S. Heidrich, S. R. Edwards, J. Dolfing, S. E. Cotterill, T. P. Curtis, Bioresour. Technol. 2014, 173, 87; L. Gil-Carrera, A. Escapa, P. Mehta, G. Santoyo, S. R. Guiot, A. Morán, B. Tartakovsky, Bioresour. Technol. 2013, 130, 584.

[28] T. Shu, D. Dang, D.-w. Xu, R. Chen, S.-j. Liao, C.-t. Hsieh, A. Su, H.-y. Song, L. Du, Electrochim. Acta. 2015, 177, 168; C. Liu, C.-C. Wang, C.-C. Kei, Y.-C. Hsueh, T.-P. Perng, Small. 2009, 5, 1535.

[29] P. Le-Clech, V. Chen, T. A. G. Fane, J. Membrane. Sci. 2006, 284, 17.

[30] J.-Y. Nam, J. C. Tokash, B. E. Logan, Int. J. Hydrogen. Energ. 2011, 36, 10550.

[31] J. Liu, L. Liu, B. Gao, F. Yang, J. Crittenden, N. Ren, Int. J. Hydrogen. Energ. 2014, 39, 17865; J. Song, L. Liu, F. Yang, N. Ren, J. Crittenden, Bioresour. Technol. 2013, 147, 409. 